\title{
Immunotherapy for thymoma
}

\author{
Marko Jakopovic ${ }^{1,2}$, Lela Bitar ${ }^{2}$, Fran Seiwerth ${ }^{2}$, Ante Marusic ${ }^{2}$, Kristina Krpina ${ }^{2}$, Miroslav Samarzija ${ }^{1,2}$ \\ ${ }^{1}$ School of Medicine, University of Zagreb, Zagreb, Croatia; ${ }^{2}$ Department for Respiratory Diseases Jordanovac, University Hospital Centre Zagreb, \\ Zagreb, Croatia \\ Contributions: (I) Conception and design: M Jakopovic, M Samarzija, A Marusic; (II) Administrative support: L Bitar, F Seiwerth; (III) Provision of \\ study materials or patients: M Jakopovic, M Samarzija, A Marusic; (IV) Collection and assembly of data: M Jakopovic, L Bitar, F Seiwerth, K Krpina; \\ (V) Data analysis and interpretation: Marko Jakopovic, Lela Bitar, Fran Seiwerth; (VI) Manuscript writing: All authors; (VII) Final approval of \\ manuscript: All authors. \\ Correspondence to: Marko Jakopovic. Department for Respiratory Diseases Jordanovac, University Hospital Centre, Jordanovac 104,10000 Zagreb, \\ Croatia. Email: marko.jakopovic@kbc-zagreb.hr.
}

\begin{abstract}
Thymic epithelial tumors (TETs) are rare thymic neoplasms. There are approximately 1.5 cases per million TETs per year. They are the most common anterior mediastinal tumors in adults. Due to limited activity of available treatment options novel strategies and treatment options are needed and treatment with immune checkpoint inhibitors is an attractive option. Thymic epithelial tumors have one of the lowest tumor mutational burden among all cancer in adults, but high expression of PD-L1 on tumor cells and abundant CD8+ lymphocytes provide a strong rational for implementing immune checkpoint inhibitors (ICIs) which target PD-1/PD-L1 pathway in the treatment of TETs. Few small early stage clinical trials were published so far evaluating efficacy of pembrolizumab and avelumab in thymoma and thymic carcinoma patients. Al trials showed reasonable response rates and progression-free survival. Higher PD-L1 expression was predictor of response in all trials. However, increased incidence of immune-related adverse events was seen in TET patients treated with immune checkpoint inhibitors compared to patients with other cancers. At the moment, ICIs are not standard of care for patients with TET and larger trials are needed to establish the right role of ICIs regarding efficacy and safety of these agents.
\end{abstract}

Keywords: Thymic epithelial tumor (TET); thymoma; thymic cancer; immune checkpoint inhibitors (ICIs); immune-related adverse events

Submitted Feb 24, 2020. Accepted for publication Jun 15, 2020.

doi: 10.21037/jtd-2019-thym-12

View this article at: http://dx.doi.org/10.21037/jtd-2019-thym-12

\section{Introduction}

Thymic epithelial tumors (TETs) are rare thymic neoplasms. There are approximately 1.5 cases per million TETs per year. They are the most common anterior mediastinal tumors in adults (1). According to the World Health Organization TETs are classified according to histology into types $\mathrm{A}, \mathrm{AB}, \mathrm{B} 1, \mathrm{~B} 2, \mathrm{~B} 3$ and thymic carcinoma (TC) with TC being the most aggressive subtype with the worst prognosis (2). Surgery is treatment of choice in patients with localized disease and in some cases is followed by radiotherapy and/or chemotherapy (3). In patients with unresectable disease treatment with chemotherapy or chemoradiotherapy is an option (3). Different chemotherapy regimens were studied and recommendations for the first line therapy are doxorubicin/cyclophosphamide/cisplatin (CAP) with or without prednisolone, cisplatin/doxorubicin/ vincristine/cyclophosphamide (ADOC) or cisplatin/ etoposide for thymoma and carboplatin/paclitaxel for TC (4). All of the before mentioned chemotherapy regimens were studied in small phase II trials or retrospectively and the results showed limited activity of chemotherapy, especially in refractory disease. Efficacy of different targeted agents was analyzed in refractory TETs such as epidermal growth factor receptor inhibitors, inhibitor of angiogenesis, histone deacetylase inhibitors, c-KIT inhibitors, octreotide and 
Src inhibitors. There was no significant benefit in relapsed TETs (5-7).

Due to limited activity of available treatment options novel strategies and treatment options are needed.

\section{Thymus}

The thymus has an important role in immune system development during childhood. Major histocompatibility complex (MHC) class II and autoimmune regulator genes (AIRE) are expressed on thymic cells surfaces, among other antigens (8). Migration of T cells through the thymic cortex and corticomedullary junction is required for maturation of $\mathrm{T}$ cells and a normally operating $\mathrm{T}$ cell receptor. During formation of $\mathrm{T}$ cells, autoreactive $\mathrm{T}$ cells which react against tissue-specific self-antigens (TSAs) undergo apoptosis, a process crucial for avoiding auto-immune intolerance (9).

In TETs, thymic epithelial cells with abnormal architecture, downregulated MHC II complex and absent AIRE form atypical formations. Consequently, autoreactive $\mathrm{T}$ cells are released into the circulation which leads to development of auto-immune disorders such as myasthenia gravis, erythroblastopenia and T/B cell immunodeficiency, called Good's syndrome when occurring with thymoma (10).

\section{Immunotherapy}

In recent years different immunotherapeutic options like cancer vaccines, immune checkpoint inhibitors (antiPD-1, anti-PD-L1, and anti-CTLA-4) and adoptive cell therapy have been extensively studied in different types of cancer. The most frequently used anti-PD-1 antibodies are nivolumab and pembrolizumab. Both of these drugs are humanized IgG4 monoclonal antibodies against PD-1 receptor. Nivolumab has been approved for treatment of the patients with renal cell and urothelial carcinoma, melanoma, non-small cell lung cancer, head and neck cancer and Hodgkin lymphoma. Pembrolizumab has been approved for treatment of the patients with head and neck cancer, urothelial carcinoma, metastatic melanoma, non-small cell lung cancer, Hodgkin lymphoma and solid tumors with high microsatellite instability. There are three anti-PD-L1 monoclonal IgG1 antibodies (atezolizumab, avelumab and durvalumab). Atezolizumab is used in treatment of the patients diagnosed with non-small cell lung cancer and urothelial carcinoma. Ipilimumab is an anti-CTLA-4 monoclonal IgG1 antibody. It is approved only for patients with metastatic melanoma (11).
Many biomarkers were analyzed as predictors of efficacy of immune checkpoint inhibitors, but only two PD-L1 expression in the tumor tissue and tumor mutational burden (TMB) were useful. The most common predictor of PD-1 and PD-L1 therapy response is high PD-L1 expression $(12,13)$.

TMB shows the number of nonsynonymous single nucleotide variants in a tumor genome coding area. Tumors with higher TMB implicitly have more neoantigens, thus enhancing immune recognition and $\mathrm{T}$-cell responsiveness which can lead to better efficacy of immune checkpoint inhibitors as it was shown in previous studies $(14,15)$.

Further, for adequate activation of immune system presence of tumor-infiltrating lymphocytes (TILs) in tumor tissue is required. Patients that have higher TILs density in tumor tissue show better treatment response (16).

\section{PD-L1 expression in TETs}

PD-L1 expression in TETs tumor cells was analyzed in limited number of studies with relatively small number of cases compared to other, more frequent types of cancer (11). The other issue is that different immunohistochemistry (IHC) antibodies were used: 15, E1L3N, EPR1161, SP142 and 28-1, as well as different cut-off values. High expression of PD-L1 was observed in $23-92 \%$ of thymoma cases, and $36-80 \%$ of thymic carcinomas (17-24). T cell recognition of antigens on TETs tumor cells and interferon-gamma (IFN- $\gamma$ ) release, leading to PD-L1 expression upregulation could be a potential explanation (25).

The same studies also tried to correlate clinicopathological factors which are associated with high expression of PD-L1. These factors are young age, higher stage (Masaoka II and IV), incomplete resection and neoadjuvant therapy in thymoma. However, conflicting results were observed regarding histology. Some authors suggested that more aggressive histology can be predictor of high expression (TC rather than thymoma), while Arbour and colleagues (23) found that high expression of PD-L1 is more likely in thymomas (17-24).

Prognostic impact of PD-L1 was also investigated and results were conflicting. Katsuya $e t a l$. and Weissferdt $e t$ al. haven't found any correlation between PD-L1 expression and outcomes $(18,22)$.

Padda and colleagues showed that patients with high PD-L1 expression have poor overall survival (OS) (17) in contrast to Arbour et al. (23) and Yokoyama et al. (20) who showed better OS in patients with high PD-L1 expression. 
These differing results are probably due to small number of cases in each study, retrospective analysis and different clinical behavior of thymomas and thymic carcinomas. Thymoma patients are known to present with autoimmune disorders like myasthenia gravis whereas TCs never present with autoimmune disorders (10). These differences in clinical presentation can be explained by different pathology findings between thymomas and TCs. Pathological findings of thymomas involve immature $\mathrm{T}$ lymphocytes whereas TCs never present with immature $\mathrm{T}$ cells, therefore never present with autoimmune disorder (2).

Thus, the prognostic significance of TETs PD-L1 expression should be taken in account with caution and further studies are needed to put significance of PD-L1 expression in correct perspective.

\section{PD-1 expression of TILs in TETs}

Some of the above-mentioned studies also investigated expression of PD-1 on TILs in TETs. Again, different cutoff values of PD-1 expression were used. Weissferdt $e t$ al. showed that PD- 1 positive TILs were found in $23 \%$ of TCs and $62 \%$ of thymomas (22). Katsuya and colleagues found that $62 \%$ of patients with thymic carcinoma showed high expression of PD-1 on TILs. Both studies haven't showed association between high PD-1 expression on TILs and outcome, so prognostic significance remains unclear. Further, some authors conclude PD-1 and PD-L1 expression are mutually exclusive $(18,21)$.

\section{Diagnostic tests for PD-L1}

In the above-mentioned studies different test for PD-L1 expression were used, so Rouquette et al. compared four different tests in patients with thymoma B3 and TC. They analyzed retrospectively 103 samples of thymoma B3 and thymic carcinoma for PD-L1 expression with four different antibodies (E1L3N, SP142, 22C3 and SP263). There was no difference in PD-L1 expression regarding antibodies that were used but patients with B3 thymoma had higher PD-L1 expression than patients with TC. $75 \%$ of antibodies targeting PD-L1 tested on the DAKO autostainer gave similar staining. When analyzing PD-L1 on immune cells there was no difference regarding B3 thymoma and TC with low concurrence between antibodies except E1L3N antibody. They have also showed that patients with B3 thymoma and high PD-L1 expression had better overall survival regardless of tumor stage (26).

\section{Tumor infiltrating lymphocytes in TETs}

For adequate response to immune checkpoint inhibitors presence of TILs in tumor are required (16). There is only limited data available about presence and importance of TILs in TETs. Recently published study analyzed PD-L1 expression and TILs in thymic epithelial tumors (24). Higuchi and colleagues analyzed TILs besides PD-L1, in 39 patients with thymomas of different subtypes and thymic carcinoma. In all TETs samples there was high CD3+ and CD8+ TILs infiltration, except for one case with $5 \%$ frequency of CD8+ expression in CD3+ lymphocytes. There was high percentage of CD8+ among the CD3+ lymphocytes in the tumors, around $82.8 \% \pm 18.3 \%$. CD4positive lymphocytes were also counted and authors found higher counts in the type B1-3 thymomas than in types A, $\mathrm{AB}$, or TC (24).

\section{Immunotherapies for TETs}

Although thymic epithelial tumors have one of the lowest tumor mutational burden among all cancer in adults (27), high expression of PD-L1 on tumor cells and abundant CD8+ lymphocytes provide a strong rational for implementing immune checkpoint inhibitors (ICIs) which target PD-1/PD-L1 pathway in the treatment of TETs.

There is only small number of published data with ICIs in TETs. All data were collected from small phase II trials in patients with refractory thymomas and thymic carcinomas.

Giaccone et al. showed overall response rate (ORR) of $22.5 \%$ and median duration of response of 22.4 months in 40 patients with recurrent TC treated with pembrolizumab. In survival analysis median progression-free survival was 4.2 months with one-year PFS $29 \%$, while median overall survival was 24.9 months with one-year OS $71 \%$ (28).

Rajan and colleagues conducted a phase I trial with avelumab in 7 patients diagnosed with thymoma and one TC patient. Almost 30\% of the patients had an objective response, while 2 patients even had partial response after one treatment application. All of these patients that had tumor shrinkage developed immune related adverse events, but only one of the non-responders. Responders had a higher absolute lymphocyte count, lower frequencies of B cells, regulatory $\mathrm{T}$ cells, conventional dendritic cells, and natural killer cells prior to therapy (29).

In a paper by Cho et al., 26 patients diagnosed with recurrent TC and 7 patients with recurrent thymoma. An ORR of $19.2 \%$ was observed among patients with TC 
Table 1 TET Immunotherapy clinical trials

\begin{tabular}{|c|c|c|c|c|c|c|}
\hline & TET in trial & Trial drug & No. of patients & ORR & mPFS (months) & mOS (months) \\
\hline Rajan et al. (29) & Thymoma & Avelumab & 7 & $29 \%$ & N/A & N/A \\
\hline Cho et al. (30) & Thymic carcinoma & Pembrolizumab & 26 & $19.2 \%$ & 9.7 & 14.5 \\
\hline Cho et al. (30) & Thymoma & Pembrolizumab & 7 & $28.6 \%$ & N/R & N/R \\
\hline
\end{tabular}

Table 2 TET Immunotherapy trials in progress

\begin{tabular}{lccc}
\hline Trial & Trial drug & TET & No. of patients currently enrolled \\
\hline NCT03076554 & Avelumab & Thymoma, TC & 8 \\
NCT03134118 & Nivolumab & Thymoma, TC & N/A \\
NCT03295227 & Pembrolizumab & Thymoma, TC & N/A \\
NCT03463460 & Pembrolizumab + Sunitinib & Thymoma, TC & N/A \\
NCT02364076 & Pembrolizumab + Epacadostat & Thymoma, TC & 45 \\
\hline
\end{tabular}

and $28.6 \%$ among those with thymoma. Median response duration was 9.7 months in TC and not reached in thymoma, with mPFS of 6.1 months for both groups. For thymic carcinoma the median OS was 14.5 months and not reached in thymoma (30) (Table 1).

In all above-mentioned trials, higher PD-L1 expression was linked with better response to treatment with immune checkpoint inhibitors, both in thymoma and TC histology. Also in all trials patients with autoimmune disorders in previous medical history were excluded (28-30).

Several early phase studies are enrolling patients investigating either monotherapy with ICIs (avelumab, trial number ID NCT03076554; nivolumab, trial number ID NCT03134118; pembrolizumab, trial number ID NCT03295227) or ICIs in combination with different agents (pembrolizumab and sunitinib, trial number ID NCT03463460; pembrolizumab and epacadostat, trial number ID NCT02364076). Results of these trials are eager awaited (Table 2).

Vaccines offers interesting treatment strategy and there are a lot of potential vaccines under development in a different types of cancers. Since TETs have low TMB resulting in limited expression of neoantigens, there are very few potential targets for developing vaccines for treatment of TETs.

So far, only anecdotal achievements were reported. Clinical response was seen in patients treated with personalized dendritic cell vaccine that targets mutation of
CDC73 gene (31) and Wilms' tumor-1 (WT-1) peptidebased vaccine $(32,33)$.

\section{Autoimmune related toxicity (irAE) in TET patients treated with immune checkpoint inhibitors}

Thymic epithelial tumors, especially thymomas, as described previously, are associated with reduced immune tolerance and therefore are highly associated with autoimmune paraneoplastic disorders which occurs between 30 to $40 \%$ at diagnosis (34). The most common one is myasthenia gravis and the risk of development of myasthenia gravis can be predicted (27). Myasthenia gravis is caused by development of auto-immune antibodies against acetylcholine receptors at the neuromuscular junction. It is also one of the most common irAE due to treatment with ICIs (28-30,34).

Among previously mentioned studies immune related adverse events (irAEs) occurred in $15 \%$ to $62 \%$ of patients treated with ICIs (28-30). Results of these studies puts TETs at higher risk of developing irAEs compared to lung cancer or other cancers.

Immune related adverse events can affect any organ or organ system (35). One of the most common reported irAE in TET patients treated with ICI was myasthenia gravis, especially when compared to other types of cancers treated with ICIs $(28,30)$. Myasthenia was reported in $3-14 \%$ of TET patients treated with pembrolizumab $(28,30)$. 
Myositis was the second most common musculoskeletal irAE which occurred in more than half of patients (4 out of 7 ) treated with avelumab (29) and $8 \%$ of patients treated with pembrolizumab (28).

Arun et al. analyzed immune adverse events in patients treated with avelumab. Higher risk for myositis had patients that had antibodies to acetylcholine receptors (antiAchR) and B cell lymphopenia. While patients without measureable anti-AchR antibodies didn't develop myositis the ones with measureable antibodies developed myositis in almost two third of the cases. Also $75 \%$ of the patients that had anti-striational antibodies detected before the start of avelumab developed myositis (29). These findings suggest that maybe we can predict which patients will develop myositis during treatment with ICIs. Due to small number of patients enrolled in the trial these conclusions should be evaluated prospectively in larger trials before firm conclusion can be made.

Myocarditis is serious adverse event in patients with TET treated with immunotherapy. It can be presented with significant symptoms or just asymptomatic isolated troponin elevation in the serum. It was reported incidence of $5 \%$ in TC patients, and up to $57 \%$ of thymoma patients treated with ICIs (28-30).

Other irAEs can also occur in TET patients but it seems that this incidence is similar to incidence of adverse events in patients with other forms of cancer treated with ICIs.

If we put all this together, it looks that thymoma patients are at greater risk of developing myocardial damage, as well as myositis and myasthenia gravis when compared to TC and other forms of cancer (9,28-30).

Another question is whether we can treat TET patients who have an active autoimmune disorder with immunotherapy? In all published trials, those patients were not included. Since the incidence of these disorders is high (30-40\% at the time of diagnosis), thought should be given to inclusion of patients with previously existing autoimmune disorders to trials, as well as minimization of exacerbation risk of these disorders with ISCIs treatment (28-30,34).

\section{Conclusions}

Thymic epithelial tumors are neoplasms which are difficult to treat, especially patients with metastatic and/or refractory disease. Immune checkpoint inhibitors which block PD-1/ PD-L1 pathway showed promising results in refractory thymomas and thymic carcinomas but with relatively high incidence of immune-related adverse events. At the moment, ICIs are not standard of care for patients with TET and larger trials are needed to establish the right role of ICIs regarding efficacy and safety of these agents.

\section{Acknowledgments}

Funding: None.

\section{Footnote}

Provenance and Peer Review: This article was commissioned by the Guest Editors (Dragana Jovanovic and Semra Bilaceroglu) for the series "Thymoma" published in fournal of Thoracic Disease. The article was sent for external peer review organized by the Guest Editors and the editorial office.

Conflicts of Interest: All authors have completed the ICMJE uniform disclosure form (available at: http://dx.doi. org/10.21037/jtd-2019-thym-12). The series "Thymoma" was commissioned by the editorial office without any funding or sponsorship. MJ reports personal fees and non-financial support from ASTRAZENECA, personal fees and non-financial support from ROCHE, personal fees and non-financial support from BRYSTOL MYERS SQUIBB, personal fees and non-financial support from MERCK SHARP \& DOHME, outside the submitted work. MS reports personal fees and non-financial support from ASTRAZENECA, personal fees and non-financial support from ROCHE, personal fees and non-financial support from BRYSTOL MYERS SQUIBB, personal fees and nonfinancial support from MERCK SHARP \& DOHME, outside the submitted work. The authors have no other conflicts of interest to declare.

Ethical Statement: The authors are accountable for all aspects of the work in ensuring that questions related to the accuracy or integrity of any part of the work are appropriately investigated and resolved.

Open Access Statement: This is an Open Access article distributed in accordance with the Creative Commons Attribution-NonCommercial-NoDerivs 4.0 International License (CC BY-NC-ND 4.0), which permits the noncommercial replication and distribution of the article with the strict proviso that no changes or edits are made and the original work is properly cited (including links to both the formal publication through the relevant DOI and the license). 
See: https://creativecommons.org/licenses/by-nc-nd/4.0/.

\section{References}

1. Engels EA. Epidemiology of thymoma and associated malignancies. J Thorac Oncol 2010;5:S260-5.

2. Travis WD, Brambilla E, Burke A, et al. WHO Classification of Tumours of the Lung, Pleura, Thymus and Heart. 4th ed. Lyon, France: International Agency for Research on Cancer, 2015:412.

3. Kelly RJ, Petrini I, Rajan A, et al. Thymic malignancies: from clinical management to targeted therapies. J Clin Oncol 2011;29:4820-7.

4. Rajan A, Giaccone G. Treatment of advanced thymoma and thymic carcinoma. Curr Treat Options Oncol 2008;9:277-87.

5. Thomas A, Rajan A, Berman A, et al. Sunitinib in patients with chemotherapy-refractory thymoma and thymic carcinoma: an open-label phase 2 trial. Lancet Oncol 2015;16:177-86.

6. Zucali PA, De Pas T, Palmieri G, et al. Phase II Study of Everolimus in Patients With Thymoma and Thymic Carcinoma Previously Treated With Cisplatin-Based Chemotherapy. J Clin Oncol 2018;36:342-9.

7. Chen Y, Gharwan H, Thomas A. Novel biologic therapies for thymic epithelial tumors. Front Oncol 2014;4:103.

8. Cheng M, Anderson MS. Thymic tolerance as a key brake on autoimmunity. Nat Immunol 2018;19:659-64.

9. Zhao C, Rajan A. Immune checkpoint inhibitors for treatment of thymic epithelial tumors: how to maximize benefit and optimize risk? Mediastinum 2019;3:35.

10. Marx A, Willcox N, Leite MI, et al. Thymoma and paraneoplastic myasthenia gravis. Autoimmunity 2010;43:413-27.

11. Yokoyama S, Miyoshi H. Thymic tumors and immune checkpoint inhibitors. J Thorac Dis 2018;10:S1509-15.

12. Herbst RS, Soria JC, Kowanetz M, et al. Predictive correlates of response to the anti-PD-L1 antibody MPDL3280A in cancer patients. Nature 2014;515:563-7.

13. Reck M, Rodríguez-Abreu D, Robinson AG, et al. Pembrolizumab versus Chemotherapy for PD-L1Positive Non-Small-Cell Lung Cancer. N Engl J Med 2016;375:1823-33.

14. Rizvi NA, Hellmann MD, Snyder A, et al. Cancer immunology. Mutational landscape determines sensitivity to PD-1 blockade in non-small cell lung cancer. Science 2015;348:124-8.

15. Gandara DR, Paul SM, Kowanetz M, et al. Blood- based tumor mutational burden as a predictor of clinical benefit in non-small-cell lung cancer patients treated with atezolizumab. Nat Med 2018;24:1441-8.

16. Galon J, Costes A, Sanchez-Cabo F, et al. Type, density, and location of immune cells within human colorectal tumors predict clinical outcome. Science 2006;313:1960-4.

17. Padda SK, Riess JW, Schwartz EJ, et al. Diffuse high intensity PD-L1 staining in thymic epithelial tumors. J Thorac Oncol 2015;10:500-8.

18. Katsuya Y, Fujita Y, Horinouchi H, et al. Immunohistochemical status of PD-L1 in thymoma and thymic carcinoma. Lung Cancer 2015;88:154-9.

19. Yokoyama S, Miyoshi H, Nishi T, et al. Clinicopathologic and Prognostic Implications of Programmed Death Ligand 1 Expression in Thymoma. Ann Thorac Surg 2016;101:1361-9.

20. Yokoyama S, Miyoshi H, Nakashima K, et al. Prognostic Value of Programmed Death Ligand 1 and Programmed Death 1 Expression in Thymic Carcinoma. Clin Cancer Res 2016;22:4727-34.

21. Marchevsky AM, Walts AE. PD-L1, PD-1, CD4, CD8 expression in neo-plastic and nonneoplastic thymus. Hum Pathol 2017;60:16-23.

22. Weissferdt A, Fujimoto J, Kalhor N, et al. Expression of PD-1 and PD-L1 in thymic epithelial neoplasms. Mod Pathol 2017;30:826-33.

23. Arbour KC, Naidoo J, Steele KE, et al. Expression of PD-L1 and other im-munotherapeutic targets in thymic epithelial tumors. PLoS One 2017;12:e0182665.

24. Higuchi R, Goto T, Hirotsu Y, et al. PD-L1 Expression and Tumor-Infiltrating Lymphocytes in Thymic Epithelial Neoplasms. J Clin Med 2019;8:1833.

25. Kim TK, Herbst RS, Chen L. Defining and Understanding Adaptive Resistance in Cancer Immunotherapy. Trends Immunol 2018;39:624-31.

26. Rouquette I, Taranchon-Clermont E, Gilhodes J et al. Immune biomarkers in thymic epithelial tumors: expression patterns, prognostic value and comparison of diagnostic tests for PD-L1. Biomark Res 2019;7:28.

27. Radovich M, Pickering CR, Felau I, et al. The Integrated Genomic Landscape of Thymic Epithelial Tumors. Cancer Cell 2018;33:244-58.

28. Giaccone G, Kim C, Thompson J, et al. Pembrolizumab in patients with thymic carcinoma: a single-arm, singlecentre, phase 2 study. Lancet Oncol 2018;19:347-55.

29. Rajan A, Heery CR, Thomas A et al. Efficacy and tolerability of anti-programmed death-ligand 1 (PD-L1) antibody (Avelumab) treatment in advanced thymoma. J 
Immunother Cancer 2019;7:269.

30. Cho J, Kim HS, Ku BM, et al. Pembrolizumab for Patients With Refractory or Relapsed Thymic Epithelial Tumor: An Open-Label Phase II Trial. J Clin Oncol 2019;37:2162-70.

31. Chen F, Zou Z, Du J, et al. Neoantigen identification strategies enable personalized immunotherapy in refractory solid tumors. J Clin Invest 2019;129:2056-70.

32. Oji Y, Inoue M, Takeda Y, et al. WT1 peptidebased immunotherapy for advanced thymic epithelial malignancies. Int J Cancer 2018;142:2375-82.

Cite this article as: Jakopovic M, Bitar L, Seiwerth F, Marusic A, Krpina K, Samarzija M. Immunotherapy for thymoma. J Thorac Dis 2020;12(12):7635-7641. doi: 10.21037/jtd-2019thym-12
33. Takahashi N, Zhao C, Rajan A. WT1 as an immunotherapy target for thymic epithelial tumors: a novel method to activate anti-tumor immunity. Mediastinum 2019;3:11.

34. Bernard C, Frih H, Pasquet F, et al. Thymoma associated with autoimmune diseases: 85 cases and literature review. Autoimmun Rev 2016;15:82-92.

35. June CH, Warshauer JT, Bluestone JA. Is autoimmunity the Achilles' heel of cancer immunotherapy? Nat Med 2017;23:540-7. 